\title{
On the existence of curves with prescribed $a$-number
}

\author{
Zijian Zhou
}

\begin{abstract}
We study the existence of Artin-Schreier curves with large $a$-number. We show that Artin-Schreier curves with large $a$-number can be written in certain forms and discuss their supersingularity. We also give a basis of the de Rham cohomology of Artin-Schreier curves. By computing the rank of the Hasse-Witt matrix of the curve, we also give bounds on the $a$-number of trigonal curves of genus 5 in small characteristic.
\end{abstract}

\section{Introduction}

Let $k$ be an algebraically closed field of characteristic $p>0$. By a curve we mean a smooth irreducible projective curve defined over $k$. Let $X$ be a curve defined over $k$ and $\operatorname{Jac}(X)$ be its Jacobian. Such a curve has several invariants, e.g. the $a$-number and the $p$-rank. The $a$-number of the curve $X$ is defined as $a_{X}=$ $\operatorname{dim}_{k}\left(\operatorname{Hom}\left(\alpha_{p}, \operatorname{Jac}(X)\right)\right)$ with $\alpha_{p}$ the group scheme which is the kernel of Frobenius on the additive group scheme $\mathbb{G}_{a}$. The $a$-number of $X$ is equal to $g-r$ where $g$ is the genus of $X$ and $r$ is the rank of the Cartier-Manin matrix, that is, the matrix for the Cartier operator defined on $H^{0}\left(X, \Omega_{X}^{1}\right)$. We refer to [3] and [14] for the properties of the Cartier operator. The $p$-rank of a curve $X$ is the number $f_{X}$ such that $\# \operatorname{Jac}(X)[p](k)=p^{f_{X}}$. One sees that $1 \leq a_{X}+f_{X} \leq g$. Moreover, a curve is called supersingular if its Jacobian is isogenous to a product of supersingular elliptic curves.

A curve $X$ of genus $g$ is called superspecial if $a_{X}=g$. Ekedahl [5] showed that for a superspecial curve $X$ one has $g \leq p(p-1) / 2$. It is known that the locus of principally polarized abelian varieties with given $a$-number $a$ has codimension

Key words and phrases: Cartier operator, Cartier-Manin matrix, Hasse-Witt matrix, ArtinSchreier curve, trigonal curve, a-number.

2010 Mathematics Subject Classification: 11G20, 15B33, 14H05, 14F40. 
$a(a+1) / 2$ in the moduli space $\mathcal{A}_{g} \otimes \mathbb{F}_{p}$ of principally polarized abelian varieties of dimension $g$, see [12, Corollary 5.4]. It is interesting to see how these loci intersect the Torelli locus of Jacobian varieties. The cases with $a=g$ or close to $g$ are here of special interest. For hyperelliptic curves with $p=2$, Elkin and Pries [7] gave a complete description of their $a$-numbers. For an Artin-Schreier curve $X$, that is, a $\mathbb{Z} / p \mathbb{Z}$-Galois cover of $\mathbb{P}^{1}$, Farnell and Pries [8] first gave non-trivial examples of families of Artin-Schreier curves with constant $a$-number. Booher and Cais [2] gave upper and lower bounds for $a$-numbers of Artin-Schreier curves.

For an Artin-Schreier curve of genus $g$ with $p=2$ and $a_{X}=g-1$, it was known that the curve has genus $g \leq 3$ [15, Corollary 3.2] and can be written as certain form [7, Theorem 1.2]; for $p \geq 3$, we show that an Artin-Schreier curve with $a$-number $g-1$ has genus $g \leq p(p-1) / 2$ and can be written as $y^{p}-y=f(x)$ with $f(x)$ a polynomial whose degree divides $p+1$, see Proposition 2.2. Moreover, we have the following.

Theorem 1.1. Let $k$ be an algebraically closed field with $\operatorname{char}(k)=p \geq 3$. Let $X$ be an Artin-Schreier curve of genus $g>0$ with equation $y^{p}-y=f(x)$. If $a_{X}=g-1$, then $f(x) \in k[x]$ and if $d=\operatorname{deg} f(x)$ then either $p=5, d=3$ and $X$ is isomorphic to a supersingular curve of genus 4 with equation

$$
y^{5}-y=x^{3}+a_{1} x, \quad a_{1} \neq 0,
$$

or $p=3, d=4$ and $X$ is isomorphic to a supersingular curve of genus 3 with equation

$$
y^{3}-y=x^{4}+a_{2} x^{2}, \quad a_{2} \neq 0 .
$$

Note that for a curve $X$ with $a_{X}=g-1$, we have $f_{X}=0$ or 1 since $a_{X}+f_{X} \leq g$. Moreover by the Deuring-Shafarevich formula [16], an Artin-Schreier curve $X$ has $p$-rank $(m-1)(p-1)$, where $m$ is the number of branch points. Hence it is not possible for Artin-Schreier curves to have $a_{X}=g-1$ and $f_{X}=1$ when $p$ is odd. We prove these results mainly by explicitly calculating the action of the Cartier operator on a basis of holomorphic differential forms. To show the supersingularity we use the de Rham cohomology.

Let $X$ be an Artin-Schreier curve with $a_{X}=g-2$. Then for $p \geq 5$, by the Deuring-Shafarevich formula the curve $X$ can be written as $y^{p}-y=f(x)$ with $f(x)$ a polynomial. For $p=3$, we give an explicit form of $X$, see Proposition 2.5. Moreover, we have the following.

Proposition 1.2. Let $X$ be an Artin-Schreier curve of genus $g>0$ given by an equation $y^{p}-y=f(x)$, where $f(x) \in k[x]$ and $\operatorname{deg} f(x)=d$. If $d \mid p+1$ and $a_{X}=g-2$, then $p=7, d=4$ and $X$ is isomorphic to the supersingular curve of genus 9 with equation

$$
y^{7}-y=x^{4}+a_{1} x, \quad a_{1} \in k^{*} .
$$


Recall that a result of $\operatorname{Re}[13]$ states that if $X$ is a non-hyperelliptic curve of genus $g$, then

$$
a_{X} \leq \frac{p-1}{p+1}\left(\frac{2 g}{p}+g+1\right)
$$

The following results improve Re's bound for trigonal curves of genus 5 in low characteristics. Note that a trigonal curve of genus 5 is not hyperelliptic; see, for example, [10, Section 2.1].

Theorem 1.3. Let $k$ be an algebraically closed field of characteristic 2 . If $X$ is a trigonal curve of genus 5 defined over $k$, then $a_{X} \leq 2$.

Theorem 1.4. Let $k$ be an algebraically closed field of characteristic 3 . If $X$ is a trigonal curve of genus 5 defined over $k$, then $a_{X} \leq 3$.

For $g=5$ and $p=2$, Re's bound says that $a_{X} \leq 3$, while our result implies that $a_{X} \leq 2$. Also for $g=5$ and $p=3$, Re's bound says that $a_{X} \leq 4$, while our result implies $a_{X} \leq 3$.

\section{On the existence of Artin-Schreier curves with prescribed a-number}

Let $\operatorname{char}(k)=p \geq 3$. Before giving the proof of Theorem 1.1, we recall and prove several results needed for Theorem 1.1 and give a basis of the de Rham cohomology for Artin-Schreier curves.

Since $a_{X}+f_{X} \leq g$, a superspecial curve has $p$-rank 0. Moreover for superspecial Artin-Schreier curves we have the following result of Irokawa and Sasaki [9].

Theorem 2.1. Let $k$ be an algebraically closed field of $\operatorname{char}(k)=p \geq 3$. Let $X$ be a superspecial Artin-Schreier curve with equation $y^{p}-y=f(x)$, where $f(x) \in k[x]$ and $\operatorname{deg} f(x)=d \geq 2$ with $\operatorname{gcd}(p, d)=1$. Then $X$ is isomorphic to a curve given by $y^{p}-y=x^{d}$ with $d \mid p+1$.

For the next step, $a_{X}=g-1$, we have the following.

Proposition 2.2. Let $k$ be an algebraically closed field with $\operatorname{char}(k)=p>0$. Let $X$ be an Artin-Schreier curve of genus $g \geq 1$. If $a_{X}=g-1$, then

(1) if $p=2$, then $g \leq 3$ and the curve $X$ can be either written as $y^{2}+y=f(x)$, where $f(x) \in k[x]$ and $\operatorname{deg} f(x)=5$ or 7 , or as $y^{2}+y=f_{0}(x)+1 / x$ with $\operatorname{deg} f_{0}(x)=1$ or 3 and $f_{0}(x) \in x k[x]$;

(2) if $p \geq 3$, then $g \leq(p-1) p / 2$ and $X$ is isomorphic to a curve with equation

$$
y^{p}-y=x^{d}+a_{d-2} x^{d-2}+\ldots+a_{1} x,
$$

where $d \mid p+1$. Moreover if $d=p+1$, then at least one of the $a_{i}$ with $2 \leq i \leq d-2$ is non-zero. If $d<p+1, d \mid p+1$, then at least one of the $a_{i}$ with $1 \leq i \leq d-2$ is non-zero. 
Proof. Part (1) of Proposition 2.2 was known, see for example [7, Theorem 1.2]. Here we only give a proof of part (2).

Suppose that $f(x)$ has poles at $\infty, Q_{1}, \ldots, Q_{m}$ for some $m \in \mathbb{Z}_{\geq 0}$. Let $x-\xi_{i}$ be a local parameter at $Q_{i}$. Write $x_{i}=1 /\left(x-\xi_{i}\right)$ for $i=1, \ldots, m$ and $x_{0}=x$. Then $f(x)$ can be written as

$$
f(x)=f_{0}(x)+\sum_{i=1}^{m} f_{i}\left(1 /\left(x-\xi_{i}\right)\right)=\sum_{i=0}^{m} f_{i}\left(x_{i}\right),
$$

where $\operatorname{deg} f_{i}(x)=d_{i}$. By $\left[17\right.$, Lemma 1], a basis of $H^{0}\left(X, \Omega_{X}^{1}\right)$ is given by $B=\cup_{s=0}^{m} B_{s}$ where

$$
\begin{aligned}
& B_{0}=\left\{x^{i} y^{j} \mathrm{~d} x \mid i, j \in \mathbb{Z}_{\geq 0}, i p+j d \leq(p-1)\left(d_{0}-1\right)-2\right\}, \\
& B_{s}=\left\{x_{s}^{i} y^{j} \mathrm{~d} x \mid i \in \mathbb{Z}_{\geq 1}, j \in \mathbb{Z}_{\geq 0}, i p+j d \leq(p-1)\left(d_{s}+1\right)\right\}, \quad s=1, \ldots, m .
\end{aligned}
$$

The condition $a_{X}=g-1$ is equivalent to the rank of the Cartier operator $\operatorname{rank}(\mathcal{C})$ being equal to 1 . Note that if $f(x)=\sum_{i=0}^{m} f_{i}\left(x_{i}\right)$ as in (3), we always have $x_{s} \mathrm{~d} x \in B$ for $1 \leq s \leq m$. Note that $\mathcal{C}\left(x_{s} \mathrm{~d} x\right) \neq 0$ and we get $\operatorname{rank}(\mathcal{C}) \geq m$.

If $p \geq 3$, then by the Deuring-Shafarevich formula, the $p$-rank of $X$ is 0 since the $a$-number of $X$ is $g-1$. We show the following:

(a) For all $p \geq 3$, we have $d \leq p+1$;

(b) If $p=5$ and $d=4$, then $\operatorname{rank}(\mathcal{C}) \geq 2$;

(c) If $p \geq 7$ and $d \geq 3$ with $d \nmid p+1$, then $\operatorname{rank}(\mathcal{C}) \geq 2$.

Then by a change of coordinates and by Theorem 2.1, one can easily prove the existence of non-zero coefficients in $f$. After excluding the cases where $\operatorname{rank}(\mathcal{C}) \geq 2$ or $\operatorname{rank}(\mathcal{C})=0$, what is left are curves with $a$-number $g-1$. Note that if $d=2$ and $f(x) \in k[x]$, the curve with equation $y^{p}-y=f(x)$ is superspecial.

By a change of coordinates, we may assume

$$
f(x)=x^{d}+a_{d-2} x^{d-2}+\ldots+a_{1} x+a_{0}, \quad d \geq 3 .
$$

Then a basis of $H^{0}\left(X, \Omega_{X}^{1}\right)$ is

$$
B=B_{0}=\left\{x^{i} y^{j} \mathrm{~d} x \mid i, j \in \mathbb{Z}_{\geq 0}, p i+j d \leq(p-1)(d-1)-2\right\} .
$$

(a) If $d \geq p+2$, then by definition we have $x^{p-1} \mathrm{~d} x \in B$. There exist $l, b \in \mathbb{Z}_{\geq 0}$ such that $d=l p+b$ with $l=1$ and $2 \leq b \leq p-1$ or $l \geq 2$ and $1 \leq b \leq p-1$. One can show $x^{p-1-b} y \mathrm{~d} x \in B$ by checking $(p-1-b) p+d \leq(p-1)(d-1)-2$. Then

$$
\begin{aligned}
\mathcal{C}\left(x^{p-1-b} y \mathrm{~d} x\right) & =\mathcal{C}\left(x^{p-1-b}\left(y^{p}-f(x)\right) \mathrm{d} x\right) \\
& =y \mathcal{C}\left(x^{p-1-b} \mathrm{~d} x\right)-\mathcal{C}\left(x^{p-1-b} f(x) \mathrm{d} x\right) \neq 0
\end{aligned}
$$

as the leading term of $x^{p-1-b} f(x)$ is $x^{l p+p-1}$. This contradiction shows that $d \leq p+1$. 
(b) For $p=5$, by $(a)$ we have $d \leq p+1$. If $d \nmid p+1$, then $d=4$ and $y^{1} \mathrm{~d} x, y^{2} \mathrm{~d} x \in B$. Additionally, we have $\mathcal{C}\left(y^{i} \mathrm{~d} x\right)=y^{i-1} \mathrm{~d} x$ for $i=1,2$ and hence $\operatorname{rank}(\mathcal{C}) \geq 2$, a contradiction. We therefore have $d \mid p+1$.

(c) For $p \geq 7$ and $d \leq p+1$, assume we have $d \nmid p+1$. Then there exists $l \in \mathbb{Z}_{>0}$ such that $l d \leq p \leq(l+1) d$. Furthermore, we have $l d \leq p-1$ and $(l+1) d \geq p+2$ as $\operatorname{gcd}(d, p)=$ 1 and $d \nmid p+1$. Then there exists $b^{\prime}$ satisfying $l d+b^{\prime}=p-1$ for $0 \leq b^{\prime} \leq d-3$.

If $d=p-1$, then $l=1, b^{\prime}=0$, we get $y \mathrm{~d} x, y^{2} \mathrm{~d} x \in B$ and $\mathcal{C}\left(y^{i} \mathrm{~d} x\right)=y^{i-1} \mathrm{~d} x$ for $i=1,2$. This implies $\operatorname{rank}(\mathcal{C}) \geq 2$, a contradiction. If $d=p-2, l=1$ and $b^{\prime}=1$, then we have $i(p-1) \leq(p-1)(p-2)-2$, which implies $x y \mathrm{~d} x, x y^{2} \mathrm{~d} x \in B$. Then $\mathcal{C}(x y \mathrm{~d} x)$ and $\mathcal{C}\left(x y^{2} \mathrm{~d} x\right)$ are linearly independent and hence $\operatorname{rank}(\mathcal{C}) \geq 2$. Now if $d \leq p-3$, we show that $x^{b^{\prime}} y^{l} \mathrm{~d} x \in B$. This is equivalent to showing $l d+b^{\prime} p \leq(p-1)(d-1)-2$. Вy substituting $b^{\prime}$ with $b^{\prime}=p-1-l d$ in the inequality, we only need to show $d(l+1)(p-$ 1) $\geq p^{2}+1$, which is clear since $(l+1) d \geq p+2$.

Now we show that $x^{b^{\prime}} y^{l} \mathrm{~d} x, x^{b^{\prime}} y^{l+1} \mathrm{~d} x \in B$. It suffices to show $l d+d+b^{\prime} p \leq$ $(p-1)(d-1)-2$. We have $l d+d+b^{\prime} p \leq(p-1)-b^{\prime}+d+b^{\prime} p$ as $b^{\prime}=p-1-l d$. Hence we only need to show

$$
d \leq\left(d-b^{\prime}-2\right)(p-1)-2
$$

Note that $b^{\prime} \leq d-3$, we have $\left(d-b^{\prime}-2\right)(p-1)-2 \geq p-3 \geq d$. Then $x^{b^{\prime}} y^{l} \mathrm{~d} x$, $x^{b^{\prime}} y^{l+1} \mathrm{~d} x \in B$ and

$$
\mathcal{C}\left(x^{b^{\prime}} y^{j} \mathrm{~d} x\right)=\sum_{t=0}^{j}(-1)^{t}\left(\begin{array}{l}
j \\
t
\end{array}\right)\left(y^{l-t}\right) \mathcal{C}\left(x^{b^{\prime}} f^{t}(x) \mathrm{d} x\right)=0, \quad j=l, l+1 .
$$

Put $t=l$, then $\left.\mathcal{C}\left(x^{b^{\prime}} f^{l}(x)\right)=\mathcal{C}\left(x^{b^{\prime}+l d}+\ldots\right) \mathrm{d} x\right) \neq 0$, which implies $\operatorname{rank}(\mathcal{C}) \geq 2$. Therefore we have $d \mid p+1$.

Now we will use the de Rham cohomology $H_{d R}^{1}(X)$ for a curve $X$ of genus $g$. Recall that this is a vector space of dimension $2 g$ provided with a non-degenerate pairing, cf. [12, Section 12]. Let $X$ be an Artin-Schreier curve over $k$ of genus $g$ with equation

$$
y^{p}-y=h(x),
$$

where $h(x) \in k[x] \backslash k$ is non-zero of degree $d$. Let $\pi: X \rightarrow \mathbb{P}^{1}$ be the $\mathbb{Z} / p$-cover. Put $U_{1}=\pi^{-1}\left(\mathbb{P}^{1}-\{0\}\right)$ and $U_{2}=\pi^{-1}\left(\mathbb{P}^{1}-\{\infty\}\right)$. For the open affine cover $\mathcal{U}=\left\{U_{1}, U_{2}\right\}$, we consider the de Rham cohomology $H_{d R}^{1}(X)$ as in [11, Section 5], i.e.

$$
H_{d R}^{1}(X)=Z_{d R}^{1}(\mathcal{U}) / B_{d R}^{1}(\mathcal{U})
$$

with $Z_{d R}^{1}(\mathcal{U})=\left\{\left(t, \omega_{1}, \omega_{2}\right) \mid t \in \mathcal{O}_{X}\left(U_{1} \cap U_{2}\right), \omega_{i} \in \Omega_{X}^{1}\left(U_{i}\right), \mathrm{d} t=\omega_{1}-\omega_{2}\right\}$ and $B_{d R}^{1}(\mathcal{U})=$ $\left\{\left(t_{1}-t_{2}, \mathrm{~d} t_{1}, \mathrm{~d} t_{2}\right) \mid t_{i} \in \mathcal{O}_{X}\left(U_{i}\right)\right\}$. 
Under the action of the Verschiebung operator $V$ on $H_{d R}^{1}(X)$, one has $V\left(H_{d R}^{1}(X)\right)=H^{0}\left(X, \Omega_{X}^{1}\right)$ and $V$ coincides with the Cartier operator on $H^{0}\left(X, \Omega_{X}^{1}\right)$.

For $1 \leq i \leq g$, put $s(x)=x h^{\prime}(x)$ with $h^{\prime}(x)$ the formal derivative of $h(x)$ and write $s(x)=s^{\leq i}(x)+s^{>i}(x)$ with $s^{\leq i}(x)$ the sum of monomials of degree $\leq i$. Then we have the following proposition.

Proposition 2.3. Let $X$ be an Artin-Schreier curve over $k$ with equation $y^{p}$ $y=h(x)$, where $h(x) \in k[x]$ and $\operatorname{deg} h(x)=d$. Then $H_{d R}^{1}(X)$ has a basis with respect to $\mathcal{U}=\left\{U_{1}, U_{2}\right\}$ consisting of the following residue classes with representatives in $Z_{d R}^{1}(\mathcal{U})$

$$
\begin{aligned}
\alpha_{i, j} & =\left[\left(0, x^{i} y^{j} \mathrm{~d} x, x^{i} y^{j} \mathrm{~d} x\right)\right], \\
\beta_{i, j} & =\left[\left(\frac{y^{p-1-j}}{x^{i+1}},-\frac{\phi_{i, j}(x, y)}{x^{i+2}} \mathrm{~d} x, \frac{(p-1-j) s^{>i+2}(x) y^{p-2-j}}{x^{i+2}} \mathrm{~d} x\right)\right],
\end{aligned}
$$

where $i, j \in \mathbb{Z}_{\geq 0}, p i+j d \leq(p-1)(d-1)-2$ and $\phi_{i, j}(x, y)=(p-1-j) s \leq^{\leq i+2}(x) y^{p-2-j}+$ $(i+1) y^{p-1-j}$.

Proof. We use the exact sequence

$$
0 \longrightarrow H^{0}\left(X, \Omega_{X}^{1}\right) \longrightarrow H_{d R}^{1}(X) \longrightarrow H^{1}\left(X, \mathcal{O}_{X}\right) \longrightarrow 0
$$

The elements $\alpha_{i, j}$ are images of $x^{i} y^{j} \mathrm{~d} x$ under the embedding of $H^{0}\left(X, \Omega_{X}^{1}\right) \rightarrow$ $H_{d R}^{1}(X)$.

Clearly, $\omega_{i, j}=x^{i} y^{j} \mathrm{~d} x$ form a basis of $H^{0}\left(X, \Omega_{X}^{1}\right)$ for $i, j \in \mathbb{Z}_{\geq 0}$ with $p i+d j \leq$ $(p-1)(d-1)-2$. On the other hand, we may identify $\mathcal{O}_{X}\left(U_{2}\right)$ with the $k$-algebra $k[x, y]$ defined by (5). Moreover, $x^{i} y^{j}$ with $i \geq 0,0 \leq j \leq p-1$ form a basis of the image of $\mathcal{O}_{X}\left(U_{2}\right)$ in $\mathcal{O}_{X}\left(U_{1} \cap U_{2}\right)$. Additionally, we have $x^{i} y^{j} \in \mathcal{O}_{X}\left(U_{1}\right)$ for $0 \leq j \leq p-1$ and $-p i \geq d j$. Then the residue classes $\left[x^{i} y^{j}\right]$ form a basis of $H^{1}\left(X, \mathcal{O}_{X}\right)$ for $i<0,0 \leq j \leq$ $p-1$ and $-p i-d j<0$. By substituting $i=-\left(i^{\prime}+1\right), j=p-1-j^{\prime}$, the residue classes $\left[x^{i+1} y^{p-1-j}\right]$ form a basis with $i \geq 0,0 \leq j \leq p-1$ and $p i+j d \leq(d-1)(p-1)-2$.

Now we check the equality that $\mathrm{d} f_{i, j}=\omega_{i, j, 1}-\omega_{i, j, 2}$ for residue classes $\beta_{i, j}=$ $\left[\left(f_{i, j}, \omega_{i, j, 1}, \omega_{i, j, 2}\right)\right]$. Note that

$$
\begin{aligned}
\mathrm{d} f_{i, j} & =\mathrm{d} \frac{y^{p-1-j}}{x^{i+1}}=\frac{(p-1-j) x^{i+1} y^{p-2-j} \mathrm{~d} y}{x^{2 i+2}}-\frac{(i+1) x^{i} y^{p-1-j} \mathrm{~d} x}{x^{2 i+2}} \\
& =\frac{-(p-1-j) x^{i+1} y^{p-2-j} h^{\prime}(x) \mathrm{d} x}{x^{2 i+2}}-\frac{(i+1) x^{i} y^{p-1-j} \mathrm{~d} x}{x^{2 i+2}} \\
& =-\frac{\phi_{i, j}(x, y) \mathrm{d} x}{x^{i+2}}-\frac{(p-1-j) y^{p-2-j} s^{>i+2}(x) \mathrm{d} x}{x^{i+2}}=\omega_{i, j, 1}-\omega_{i, j, 2},
\end{aligned}
$$

which ends the proof. 
Remark 2.4. The pairing $\langle$,$\rangle for this basis is as follows: \left\langle\alpha_{i_{1}, j_{1}}, \beta_{i_{2}, j_{2}}\right\rangle \neq 0$ if $\left(i_{1}, j_{1}\right)=\left(i_{2}, j_{2}\right)$ and $\left\langle\alpha_{i_{1}, j_{1}}, \beta_{i_{2}, j_{2}}\right\rangle=0$ otherwise. Indeed, for $\left(i_{1}, j_{1}\right)=\left(i_{2}, j_{2}\right)$ we have ord $\cos _{\infty}\left(y^{p-1} / x \mathrm{~d} x\right)=-1$ and hence $\left\langle\alpha_{i_{1}, j_{1}}, \beta_{i_{2}, j_{2}}\right\rangle \neq 0$. For other cases, the proof is similar to the proof of [18, Theorem 4.2.1].

\subsection{Proof of Theorem 1.1}

Note that by Proposition $2.2(2)$, curve $X$ can be written as certain form with $\operatorname{rank}(\mathcal{C})=1$. For $d \leq 2$, the situation is trivial and $\operatorname{rank}(\mathcal{C})=0$ for all $p>0$. Then we may assume that the polynomial $f(x)$ has the form:

$$
f(x)=x^{d}+a_{d-2} x^{d-2}+\ldots+a_{1} x, \quad d>2 .
$$

Also a basis of $H^{0}\left(X, \Omega_{X}^{1}\right)$ is given by forms below:

$$
B=\left\{x^{i} y^{j} \mathrm{~d} x \mid i p+j d \leq(p-1)(d-1)-2\right\} .
$$

(1) For $p \geq 7$, we show that $\operatorname{rank}(C) \neq 1$. Indeed, if $a_{i}=0$ for $i \in\{1,2, \ldots, d-2, d\}$, then by Theorem 2.1 we have $\operatorname{rank}(\mathcal{C})=0$. Otherwise, let $i_{0}$ be the largest integer in $\{1,2, \ldots, d-2\}$ such that $a_{i_{0}} \neq 0$. There are non-negative integers $l, m, b$ satisfying $l d=p+1$ and $d-2=m i_{0}+b$ with $b \leq i_{0}-1$.

Suppose $2 \leq i_{0} \leq d-2$, we show that $x^{b} y^{l-1+m} \mathrm{~d} x \in B$. This is equivalent to showing

$$
b p+(l+m-1) d \leq(d-1)(p-1)-2,
$$

for $m \geq 1, i_{0} \geq 2$. By substituting $b=d-2-m i_{0}$, one can show this is equivalent to $m\left(p i_{0}-d\right) \geq 2$, which is trivial as $d \mid p+1$ and $m\left(p i_{0}-d\right) \geq 2 p-d \geq 2$.

Now if $d=p+1$, then we have $l=1$ and $x^{b} y^{m} \mathrm{~d} x \in B$ as showed above. If $b=0$, we have $d-2=p-1=m i_{0}$. By $i_{0} \geq 2$, we have $m \leq(p-1) / 2$. We show that $y^{m+1} \mathrm{~d} x \in B$ if $p \geq 5$. It is sufficient to show that $(m+1) \leq(p-1)(d-1)-2=p(p-1)-2$. This is true for $p \geq 5$. Then $\mathcal{C}\left(y^{m+1} \mathrm{~d} x\right) \neq 0$ and $\mathcal{C}\left(y^{m} \mathrm{~d} x\right) \neq 0$ are linearly independent. This $\operatorname{implies} \operatorname{rank}(\mathcal{C}) \geq 2$ for $b=0$. Suppose $b \geq 1$. We show that $x^{b-1} y^{m+1} \in B$. Note that $d-2=p-1=m i_{0}+b$. By a similar fashion, we only need to show $m\left(i_{0}-1\right)(p-1) \geq 4$, which is true if $p \geq 5$. Then

$$
\begin{aligned}
\omega_{b, m}:=\mathcal{C}\left(x^{b} y^{m} \mathrm{~d} x\right)=\mathcal{C}\left(x^{b}\left(y^{p}-f(x)\right)^{m} \mathrm{~d} x\right) & =\mathcal{C}\left((-1)^{m} x^{b} a_{i_{0}}^{m}\left(x^{i_{0}}\right)^{m} \mathrm{~d} x\right)+\ldots \\
& =\mathcal{C}\left((-1)^{m} a_{i_{0}}^{m} x^{p-1} \mathrm{~d} x\right)+\ldots \neq 0 .
\end{aligned}
$$

Similarly, we have

$$
\begin{aligned}
\omega_{b-1, m+1}: & =\mathcal{C}\left(x^{b-1} y^{m+1} \mathrm{~d} x\right)=\mathcal{C}\left(x^{b-1}\left(y^{p}-f(x)\right)^{m+1} \mathrm{~d} x\right) \\
& =\mathcal{C}\left((m+1)(-1)^{m+1} a_{p+1} a_{i_{0}}^{m} x^{2 p-1} \mathrm{~d} x\right)+\ldots \neq 0 .
\end{aligned}
$$

Since $\omega_{b, m}$ and $\omega_{b-1, m+1}$ are $k$-linearly independent, we have $\operatorname{rank}(\mathcal{C}) \geq 2$. 
If $d \mid p+1$ and $d \leq(p+1) / 2$, we show that $x^{b} y^{l+m} \mathrm{~d} x \in B$, which is equivalent to $b p+(l+m) d \leq(d-1)(p-1)-2$. Since $m\left(p i_{0}-d\right) \geq 2 p-d$, we only need to show $m\left(p i_{0}-d\right)-d \leq 2$, which is true for $p \geq 7$. Hence $\mathcal{C}\left(x^{b} y^{l+m} \mathrm{~d} x\right) \neq 0$ and $\mathcal{C}\left(x^{b} y^{l+m-1} \mathrm{~d} x\right) \neq 0$ by the same method above.

Assume $i_{0}=1$ and $a_{i}=0$ for any $i \in 2,3, \ldots, d-2$, if $d=p+1$, by a simple change of coordinates and by Theorem 2.1 the curve is superspecial and $\operatorname{rank}(\mathcal{C})=0$. Otherwise we have $d<p+1$, in this case we have $d-2=m+b$. We show that $y^{l+m+b-1} \mathrm{~d} x$, $y^{l+m+b} \mathrm{~d} x \in B$, which is equivalent to showing

$$
(l+m+b-1) d \leq(d-1)(p-1)-2 \quad \text { and } \quad(l+m+b) d \leq(d-1)(p-1)-2,
$$

respectively. These can be simplified to

$$
d^{2}-(p+2) d+2 p+2 \leq 0, \quad d^{2}-(p+1) d+2 p+2 \leq 0 .
$$

These two inequalities hold for $p \geq 11$. For $p=7$, we have $d \mid p+1=8$ and hence $d \geq 4$. Then those two inequalities also hold.

Moreover, we have

$$
\begin{aligned}
\mathcal{C}\left(y^{l+m+b-1} \mathrm{~d} x\right) & =\mathcal{C}\left(\left(y^{p}-f(x)\right)^{l+m+b-1} \mathrm{~d} x\right) \\
& =\mathcal{C}\left((-1)^{l+m+b-1}\left(x^{d}\right)^{l-1}\left(a_{1} x\right)^{m+b} \mathrm{~d} x\right)+\ldots \\
& =\mathcal{C}\left((-1)^{l+m+b-1} a_{1}^{m+b} x^{p-1} \mathrm{~d} x\right)+\ldots \neq 0
\end{aligned}
$$

and $\mathcal{C}\left(y^{l+m+b} \mathrm{~d} x\right)=\mathcal{C}\left((-1)^{l+m+b-1} a_{1}^{m+b} x^{p-1} y^{p} \mathrm{~d} x\right)+\ldots \neq 0$. Then $\operatorname{rank}(\mathcal{C}) \geq 2$.

(2) For $p=5$ and $d=p+1=6$, to get $\operatorname{rank}(\mathcal{C})=1$ we must have $i_{0} \geq 2$, otherwise $X$ is superspecial by Theorem 2.1. Then $x^{b-1} y^{m+1} \mathrm{~d} x, x^{b} y^{m} \mathrm{~d} x \in B$ for $b \geq 1$ and $y^{m+1} \mathrm{~d} x, y^{m} \mathrm{~d} x \in B$ for $b=0$ (similar to the case $p=7$ ). This implies $\operatorname{rank}(\mathcal{C}) \geq 2$. As for $d=3$, if $a_{d-2}=a_{1}=0$, then it is superspecial by Theorem 2.1. If $a_{1} \neq 0$, then $y^{2} \mathrm{~d} x \in B$ and $\operatorname{rank}(\mathcal{C})=1$.

For the supersingularity, let $X$ be a curve given by equation $y^{5}-y=x^{3}+a_{1} x$ with $a_{1} \neq 0$. Then we have $H^{0}\left(X, \Omega_{X}^{1}\right)=\left\langle\mathrm{d} x, x \mathrm{~d} x, y \mathrm{~d} x, y^{2} \mathrm{~d} x\right\rangle$ and $\mathcal{C}\left(H^{0}\left(X, \Omega_{X}^{1}\right)\right)=$ $\langle\mathrm{d} x\rangle$. Moreover by using Proposition 2.3, one can compute that $X$ has EkedahlOort type $[4,3,2]$ and the curve $X$ is supersingular by [4, Step 2, p. 1379]. For the definition of Ekedahl-Oort type we refer [6].

(3) For $p=3$, if $d=2$ the curve is superspecial. If $d=4$, then we may assume that $a_{2} \neq 0$ in $f(x)$, otherwise by a simple change of coordinates we may assume the curve is given by equation $y^{3}-y=a_{4} x^{4}$, which is superspecial by the Theorem 2.1.

If $a_{2} \neq 0$, then by a change of coordinate we get $f(x)=x^{4}+a_{2} x^{2}$. A basis of $H^{0}\left(X, \Omega_{X}^{1}\right)$ is $\{\mathrm{d} x, x \mathrm{~d} x, y \mathrm{~d} x\}$ with $\mathcal{C}(\mathrm{d} x)=\mathcal{C}(x \mathrm{~d} x)=0$ and $\mathcal{C}(y \mathrm{~d} x)=\mathcal{C}\left(-a_{2} x^{2} \mathrm{~d} x\right)=$ $-a_{2}^{1 / 3} \mathrm{~d} x \neq 0$. This implies $\operatorname{rank}(\mathcal{C})=1$. Similarly using Proposition 2.3, a curve given by equation $y^{3}-y=x^{4}+a_{2} x^{2}$ with $a_{2} \neq 0$ has Ekedahl-Oort type [3,2] and hence is supersingular by [4, Step 2, p. 1379]. 


\subsection{Proof of Proposition 1.2}

Let $X$ be an Artin-Schreier curve given by equation $y^{p}-y=f(x)$ with $\operatorname{deg} f(x)=$ $d \mid p+1$ and $\operatorname{rank}(\mathcal{C})=2$. We may assume that the polynomial $f(x)$ has the form:

$$
f(x)=x^{d}+a_{d-2} x^{d-2}+\ldots+a_{1} x .
$$

By the proof of Theorem 1.1, there is an integer $n \in\{1,2, \ldots, d-2\}$ such that $a_{n} \neq 0$. Again denote by $i_{0}$ the largest integer in $\{1,2, \ldots, d-2\}$ such that $a_{i_{0}} \neq 0$ and let $l, m, b$ be the same as in the proof of Theorem 1.1 .

For $p \geq 7$, if $d=p+1$, we show that in this case $\operatorname{rank}(\mathcal{C}) \geq 3$. Indeed by Theorem 2.1 , we have $i_{0} \geq 2$ and $d-2=p-1=m i_{0}+b$. If $b=0$, then $d-2=p-1=m i_{0}$ and $m \leq(p-1) / 2$. Moreover from the proof of Theorem 1.1, part (1), we have $y^{m} \mathrm{~d} x, y^{m+1} \mathrm{~d} x \in B$. We show that $y^{m+2} \mathrm{~d} x \in B$. It suffices to show that $(m+2) d \leq$ $(p-1)(d-1)-2$, which is equivalent to showing $(m+2)(p+1) \leq p^{2}-p-2$ for any $1 \leq m \leq(p-1) / 2$. This is true for $p \geq 7$. On the other hand, note that $\mathcal{C}\left(y^{m} \mathrm{~d} x\right)$, $\mathcal{C}\left(y^{m+1} \mathrm{~d} x\right)$ and $\mathcal{C}\left(y^{m+2} \mathrm{~d} x\right)$ are linearly independent. Then $\operatorname{rank}(\mathcal{C}) \geq 3$ in this case. Now if $b \geq 1$, we showed that $x^{b} y^{m} \mathrm{~d} x, x^{b-1} y^{m+1} \mathrm{~d} x \in B$. By a similar argument as in the case $b=0$ above, one can show that $x^{b} y^{m+1} \mathrm{~d} x \in B$. Additionally, $\mathcal{C}\left(x^{b} y^{m} \mathrm{~d} x\right), \mathcal{C}\left(x^{b-1} y^{m+1} \mathrm{~d} x\right)$ and $\mathcal{C}\left(x^{b} y^{m+1} \mathrm{~d} x\right)$ are linearly independent. Then we have $\operatorname{rank}(\mathcal{C}) \geq 3$ for $p \geq 7$ and $d=p+1$.

Now if $d \mid p+1$ and $d<p+1$, then $l=(p+1) / d \geq 2$. If $i_{0} \geq 2$, we show that $\operatorname{rank}(\mathcal{C}) \geq$ 3 for $p \geq 7$. Note that we have $x^{b} y^{l+m-1} \mathrm{~d} x, x^{b} y^{l+m} \mathrm{~d} x \in B$ by the part (1) of the proof of Theorem 1.1. We now claim that $x^{b} y^{l+m+1} \mathrm{~d} x \in B$. By definition of $B$, it suffices to show

$$
(l+m+1) d+b p \leq(p-1)(d-1)-2 .
$$

By substituting $b=d-2-m i_{0}$ and $p=l d-1$, the inequality can be simplified to $\left(i_{0} l-1\right) m \geq 3$. This is true as $i_{0} \geq 2, l \geq 2$ and $m \geq 1$. For $i_{0}=1$, we show that $\operatorname{rank}(\mathcal{C}) \geq 3$ for $p \geq 11$. Note that in this case we have $d-2=m$. One can easily show that $y^{l+m} \mathrm{~d} x, y^{l+m-1} \mathrm{~d} x \in B$ by the definition of $B$. Additionally, we show that $y^{l+m+1} \mathrm{~d} x \in B$ for $p \geq 11$. Indeed, it suffices to show $(l+m+1) d \leq(p-1)(d-1)-2$, which can be simplified to $2 l+d \leq p$. Note that $l d=p+1$, we only need to show $2(p+1) / d+d \leq p$ which can be rewritten as $d^{2}-d p+2(p+1) \leq 0$. This is true for $3 \leq d \leq(p+1) / 2$.

For $p=7$ and $i_{0}=1$, we have $d=4$ and the curve is given by equation $y^{7}-y=$ $x^{4}+a_{1} x$ with $a_{1} \in k^{*}$. Then

$$
B=\left\{x^{i} y^{j} \mathrm{~d} x, \mid i, j \in \mathbb{Z}_{\geq 0}, 7 i+4 j \leq 16\right\}
$$

and $\mathcal{C}\left(x^{i} y^{j} \mathrm{~d} x\right)=0$ for all $i, j$ except $(i, j)=(0,4),(0,3),(1,2)$. Moreover, $\mathcal{C}\left(y^{4} \mathrm{~d} x\right)$ and $\mathcal{C}\left(y^{3} \mathrm{~d} x\right)$ are linearly independent and $\mathcal{C}\left(y^{3} \mathrm{~d} x\right)=\xi \mathcal{C}\left(x y^{2} \mathrm{~d} x\right)$ for some $\xi \in k^{*}$. 
Then $\operatorname{rank}(\mathcal{C})=2$. Using Proposition 2.3 and by [4, Step 2, p. 1379] as above, the curve is supersingular.

Now let $p=5$. If $d=3$, then by Theorem 2.1 and Theorem 1.1 , we have $a_{X}=g$ or $g-1$. For $d=6$, we get $d-2=4=m i_{0}+b$. Additionally for $i_{0}=2,3,4$, one can easily show that $y^{2} \mathrm{~d} x, y^{3} \mathrm{~d} x, x y^{3} \mathrm{~d} x \in B$ and $\mathcal{C}\left(y^{2} \mathrm{~d} x\right), \mathcal{C}\left(y^{3} \mathrm{~d} x\right)$ and $\mathcal{C}\left(x y^{2} \mathrm{~d} x\right)$ are linearly independent. Hence $\operatorname{rank}(\mathcal{C}) \geq 3$ and $a_{X} \leq g-3$ with $g=10$.

For $p=3$ and $d \mid p+1=4$, by Theorem 2.1 and Theorem 1.1 , we have $a_{X} \geq g-1$.

Similar to Proposition 2.2, we have the following.

Proposition 2.5. Let $k$ be an algebraically closed field with $\operatorname{char}(k)=p \geq 3$. Let $X$ be an Artin-Schreier curve of genus $g \geq 1$ with equation $y^{p}-y=f(x)$, where $f(x) \in k(x)$. If $a_{X}=g-2$, then

(1) if $p=3$, then $g \leq 7$ and the curve $X$ can be either written as $y^{3}-y=f(x)$, where $f(x) \in k[x]$ and $\operatorname{deg} f(x) \leq 8$, or as $y^{3}-y=f_{0}(x)+f_{1}(1 / x)$ with $f_{0}(x), f_{1}(x) \in$ $k[x]$ and $\operatorname{deg} f_{0}(x) \leq 4, \operatorname{deg} f_{1}(x) \leq 2$;

(2) if $p \geq 5$, then $g \leq(2 p+1)(p-1) / 2$ and $X$ is isomorphic to a curve with equation

$$
y^{p}-y=f(x), \quad f(x) \in k[x] .
$$

The proof of part (1) is similar to the part (1) of the proof of Proposition 2.2 and hence we omit it. For part (2), one can first show $f$ is a polynomial using the Deuring-Shafarevich formula and then prove the proposition by analysing the degree of $f$.

\section{On the existence of trigonal curves with prescribed $a$-number}

Now we study the existence of trigonal curves with prescribed $a$-number and give proofs of Theorems 1.3 and 1.4. We deal here with genus 5. It is well known that a trigonal curve $X$ of genus 5 is a normalization of a quintic curve $C$ in $\mathbb{P}^{2}$ with a unique singular point [1, Exercise I-6, p. 279], see also [10, Lemma 2.2.1].

\subsection{Set up}

For a trigonal curve $X$ of genus 5 defined over $k$, let $\phi: X \rightarrow \mathbb{P}^{1}$ be a morphism of degree 3. Then using the base point free pencil trick and Clifford Theorem one can easily show that $\phi$ is unique (up to isomorphism of $\mathbb{P}^{1}$ ) and $X$ is not hyperelliptic.

Lemma 3.1. Let $p$ be either 2 or 3 . If $X$ is a trigonal curve of genus 5 over $k$, then 
(1) $X$ is a normalization of a quintic curve $C$ in $\mathbb{P}^{2}$ with a unique singular point of multiplicity 2. Moreover,

(i) If $C$ has a node, then $C$ is given by a homogeneous polynomial $F \in k[x, y, z]$ of degree 5 with

$$
F=x y z^{3}+f
$$

where $f$ is a sum of monomials not divisible by $z^{3}$.

(ii) If $C$ has a cusp, then $C$ is given by a homogeneous polynomial $F \in$ $k[x, y, z]$ of degree 5 with

$$
F=x^{2} z^{3}+f,
$$

where $f$ is a sum of monomials not divisible by $z^{3}$ and the coefficient of $y^{3} z^{2}$ in $f$ is non-zero.

(2) The normalization of any $C$ with one singular point in $(i)$ and $(i i)$ is a trigonal curve of genus 5 .

Proof. Kudo and Harashita proved the lemma for $p \neq 2$ in [10, Lemma 2.2.1] (note that we can assume $\varepsilon=0$ in the statement of Lemma 2.2.1 in [10] since $k$ is algebraically closed). For $p=2$, we show that part (1) is true and since the proof of the other part is similar to the case $p \geq 3$ we omit it.

Assuming the singular point is (0:0:1), the curve $C$ is given by $F=Q z^{3}+f$, where $Q$ is a quadratic form in $k[x, y]$ and $f$ is a sum of monomials in $x, y$ of degree $>2$.

If $Q$ is non-degenerate, then $C$ has a node. We may change coordinates so that $Q$ equals $x y$, we arrive at $F=x y z^{3}+f$ with $f$ a sum of monomials in $x, y$ of degree $>2$.

If $Q$ is degenerate, then $C$ has a cusp. We may change coordinates so that $Q$ equals $x^{2}$, we arrive at $F=x^{2} z^{3}+f$ with $f$ a sum of monomials in $x, y$ of degree $>2$.

We recall the following proposition.

Proposition 3.2. ([10, Proposition 2.3.1]) Let $X$ be a trigonal curve of genus 5 defined over $k$. Let $C$ be an associated quintic curve in $\mathbb{P}^{2}$ given by Lemma 3.1. Let $h_{l, m}(1 \leq l, m \leq 5)$ be the coefficient of the monomial $x^{p i_{l}-i_{m}} y^{p j_{l}-j_{m}} z^{p k_{l}-k_{m}}$ in $F^{p-1}$, where

\begin{tabular}{cccccc}
$l$ & 1 & 2 & 3 & 4 & 5 \\
\hline$i_{l}$ & 3 & 1 & 2 & 2 & 1 \\
$j_{l}$ & 1 & 3 & 2 & 1 & 2 \\
$k_{l}$ & 1 & 1 & 1 & 2 & 2
\end{tabular}.

Then the Hasse-Witt matrix $H$ of $X$ is given by $H=\left(h_{l, m}\right)$. 


\subsection{The proof of Theorem 1.3}

Let $p=2$ and $X$ be a trigonal curve of genus 5 defined over $k$. we start by simplifying the defining equation of the singular model $C \subset \mathbb{P}^{2}$ of $X$.

Lemma 3.3. Let $k$ be an algebraically closed field with $\operatorname{char}(k)=2$. In the notation of Lemma 3.1 case $(i)$, we can choose $f$ as

$$
f=\left(x^{3}+b_{1} y^{3}\right) z^{2}+\sum_{i=1}^{5}\left(a_{i} x^{5-i} y^{i-1}\right) z+\sum_{i=6}^{11} a_{i} x^{11-i} y^{i-6}
$$

or

$$
f=\sum_{i=1}^{5}\left(a_{i} x^{5-i} y^{i-1}\right) z+\sum_{i=6}^{11} a_{i} x^{11-i} y^{i-6}
$$

For case (ii), we can choose $f$ as

$$
f=y^{3} z^{2}+\sum_{i=1}^{5}\left(a_{i} x^{5-i} y^{i-1}\right) z+\sum_{i=6}^{11} a_{i} x^{11-i} y^{i-6} .
$$

Proof. For the case $(i)$ of Lemma 3.1, the curve $C$ is given by $F=x y z^{3}+f$, where $f$ is the sum of monomials, which have degree $>2$ in $x, y$. By a linear transformation $z \mapsto z+\alpha x+\beta y$, we may assume the coefficients of $x^{2} y z^{2}$ and $x y^{2} z^{2}$ are zero. Then

$$
f=\left(b_{0} x^{3}+b_{1} y^{3}\right) z^{2}+\sum_{i=1}^{5} a_{i} x^{5-i} y^{i-1} z+\sum_{i=6}^{11} a_{i} x^{11-i} y^{i-6},
$$

where $b_{0}, b_{1}, a_{1}, \ldots, a_{11} \in k$. Note that if $\left(b_{0}, b_{1}\right) \neq(0,0)$, by symmetry we may assume $b_{0} \neq 0$. By scaling $x \mapsto \alpha x, y \mapsto \beta y$ with $\alpha \beta=1$ and $\alpha^{3}=1$, we may assume $b_{0}=1$. On the other hand, if $b_{0}=b_{1}=0$ in $f$, then we have

$$
f=\sum_{i=1}^{5} a_{i} x^{5-i} y^{i-1} z+\sum_{i=6}^{11} a_{i} x^{11-i} y^{i-6} .
$$

For the case $(i i)$ of Lemma 3.1, the curve $C$ is given by $F=x^{2} z^{3}+f$, where $f$ is the sum of monomials, which have degree $>2$ in $x, y$ and the coefficient of $y^{3} z^{2}$ is non-zero. Consider $y \mapsto y+\gamma x$ and then consider $z \mapsto z+\alpha x+\beta y$, we may assume the coefficients of $x^{3} z^{2}, x^{2} y z^{2}$ and $x y^{2} z^{2}$ are zero. Moreover, by scaling $y \mapsto \delta y$ with $\delta^{3}=1$, we may assume the coefficient of $y^{3} z^{2}$ is equal to 1 . Then we have

$$
f=y^{3} z^{2}+\sum_{i=1}^{5} a_{i} x^{5-i} y^{i-1} z+\sum_{i=6}^{11} a_{i} x^{11-i} y^{i-6}, \quad a_{1}, \ldots, a_{11} \in k .
$$

Now we can give a proof of Theorem 1.3. 
Proof of Theorem 1.3. Let $C$ be a singular model of $X$ given by Lemma 3.1. If $C$ has a node, then by Lemma $3.3, f$ is either given by (8) or (9). If $f$ is given by (8), then by Proposition 3.2, the Hasse-Witt matrix $H$ of $X$ is equal to

$$
\left(\begin{array}{ccccc}
a_{2} & 0 & a_{1} & a_{7} & a_{6} \\
0 & a_{4} & a_{5} & a_{11} & a_{10} \\
a_{4} & a_{2} & a_{3} & a_{9} & a_{8} \\
1 & 0 & 0 & 0 & 1 \\
0 & 1 & 0 & b_{1} & 0
\end{array}\right)
$$

Let $e_{i}$ be the $i$-th row of $H$. Then $e_{4}$ and $e_{5}$ are linearly independent and $\operatorname{rank}(H) \geq$ 2 .

Now we show $\operatorname{rank}(H) \geq 3$ in this case. Indeed, if $\operatorname{rank}(H)=2$, then $e_{i}$ for $i=1,2,3$ is a linear combination of $e_{4}$ and $e_{5}$. By the shape of $H$, we have

$$
a_{1}=a_{3}=a_{5}=a_{7}=a_{10}=0, \quad a_{4}=a_{8}, \quad a_{2}=a_{6}, \quad b_{1} a_{4}=a_{11}, \quad b_{1} a_{2}=a_{9} .
$$

Hence $C$ is given by

$$
\begin{aligned}
F & =x y z^{3}+\left(x^{3}+b_{1} y^{3}\right) z^{2}+\left(a_{2} x^{3} y+a_{4} x y^{3}\right) z+a_{2} x^{5}+a_{4} x^{3} y^{2}+b_{1} a_{2} x^{2} y^{3}+b_{1} a_{4} y^{5} \\
& =\left(z+a_{2}^{1 / 2} x+a_{4}^{1 / 2} y\right)^{2}\left(b_{1} y^{3}+x^{3}+x y z\right)
\end{aligned}
$$

and $C$ is reducible. This contradiction shows that $\operatorname{rank}(H) \geq 3$.

Now if $f$ is given by (9), then again by Proposition 3.2 the Hasse-Witt matrix $H$ of $X$ is equal to

$$
\left(\begin{array}{ccccc}
a_{2} & 0 & a_{1} & a_{7} & a_{6} \\
0 & a_{4} & a_{5} & a_{11} & a_{10} \\
a_{4} & a_{2} & a_{3} & a_{9} & a_{8} \\
1 & 0 & 0 & 0 & 0 \\
0 & 1 & 0 & 0 & 0
\end{array}\right)
$$

Then we have $\operatorname{rank}(H) \geq 2$. Moreover, if $\operatorname{rank}(H)=2$, then we have $a_{i}=0$ for all $i \in\{1, \ldots, 11\}$ with $i \neq 2,4$. This implies

$$
F=x y z^{3}+a_{2} x^{3} y z+a_{4} x y^{3} z=x y\left(z^{3}+a_{2} x^{2} z+a_{4} y^{2}\right),
$$

a contradiction. Hence we have $\operatorname{rank}(H) \geq 3$ if $C$ has a node.

If the curve $C$ has a cusp, then by Lemma 3.3, $f$ is given by (10). Hence by Proposition 3.2, the Hasse-Witt matrix of $X$ is equal to

$$
\left(\begin{array}{ccccc}
a_{2} & 0 & a_{1} & a_{7} & a_{6} \\
0 & a_{4} & a_{5} & a_{11} & a_{10} \\
a_{4} & a_{2} & a_{3} & a_{9} & a_{8} \\
0 & 0 & 1 & 0 & 0 \\
0 & 0 & 0 & 1 & 0
\end{array}\right)
$$


Then we still have $\operatorname{rank}(H) \geq 2$. We show $\operatorname{rank}(H) \geq 3$ by showing that $C$ has at least two singular points if $\operatorname{rank}(H)=2$. Indeed, suppose $\operatorname{rank}(H)=2$. By (13), we obtain $a_{2}=a_{4}=a_{6}=a_{8}=a_{10}=0$. This implies

$$
F=x^{2} z^{3}+y^{3} z^{2}+\left(a_{1} x^{4}+a_{3} x^{2} y^{2}+a_{5} y^{4}\right) z+a_{7} x^{4} y+a_{9} x^{2} y^{3}+a_{11} y^{5} .
$$

Denote by $F_{x}$ (resp. $F_{y}, F_{z}$ ) the formal partial derivative with respect to the variable $x$ (resp. $y, z$ ). Note that we have

$$
F_{x}=0, F_{y}=y^{2} z^{2}+a_{7} x^{4}+a_{9} x^{2} y^{2}+a_{11} y^{4}, \quad F_{z}=x^{2} z^{2}+a_{1} x^{4}+a_{3} x^{2} y^{2}+a_{5} y^{4} .
$$

By setting $x=1$ in $F_{x}, F_{y}$ and $F_{z}$, one can easily show that $(1: a: b)$ is a singular point. Then there are at least two singular points on $C$. By the genus formula for plane curves, the genus of $X$ is less than 5 , a contradiction.

Now we have $\operatorname{rank}(H) \geq 3$ for any trigonal curve $X$ of genus 5 over $k$. Then $a_{X} \leq 2$.

\subsection{The proof of Theorem 1.4}

Let $p=3$ and $X$ be a trigonal curve of genus 5 defined over $k$. We now give the reductions of the defining equations of the singular model $C \subset \mathbb{P}^{2}$ of $X$ given by Lemma 3.1.

Lemma 3.4. Let $k$ be an algebraically closed field with $\operatorname{char}(k)=3$. In the notation of Lemma 3.1 case $(i)$, we can choose $f$ as

$$
\left(b_{0} x^{3}+b_{1} y^{3}+b_{2} x^{2} y+b_{3} x y^{2}\right) z^{2}+\sum_{i=1}^{5} a_{i} x^{5-i} y^{i-1} z+a_{6} x^{5}+\sum_{i=8, i \neq 10}^{11} a_{i} x^{11-i} y^{i-6}
$$

For the case (ii), we can choose $f$ as

$$
\left(y^{3}+\sum_{i=2}^{3} b_{i} x^{4-i} y^{i-1}\right) z^{2}+\sum_{i=1}^{5} a_{i} x^{5-i} y^{i-1} z+a_{7} x^{4} y+a_{8} x^{3} y^{2}+a_{10} x y^{4}+a_{11} y^{5}
$$

Proof. If $C$ has a node, then by Lemma $3.1, F=x y z^{3}+f$ with $f$ the sum of monomials, which have degree $>2$ in $x, y$. By a linear transform $z \mapsto z+\alpha x+\beta y$ we may assume the coefficients of $x^{4} y$ and $x y^{4}$ is zero. Then $f$ is equal to

$$
\left(b_{0} x^{3}+b_{1} y^{3}+b_{2} x^{2} y+b_{3} x y^{2}\right) z^{2}+\sum_{i=1}^{5} a_{i} x^{5-i} y^{i-1} z+a_{6} x^{5}+a_{8} x^{3} y^{2}+a_{9} x^{2} y^{3}+a_{11} y^{5} .
$$


By Lemma 3.1, if $C$ has a cusp, then $F=x y z^{3}+f$ with $f$ the sum of monomials, which have degree $>2$ in $x, y$ and the coefficient of $y^{3} z^{2}$ is non-zero. By a linear transform $z \mapsto z+\alpha x+\beta y$, we may assume the coefficients of $x^{5}$ and $x^{2} y^{3}$ is zero. Moreover, by scaling $y \mapsto \delta y$, we may assume the coefficient of $y^{3} z^{2}$ is 1 . Then we have

$$
f=\left(y^{3}+b_{2} x^{2} y+b_{3} x y^{2}\right) z^{2}+\sum_{i=1}^{5} a_{i} x^{5-i} y^{i-1} z+a_{7} x^{4} y+a_{8} x^{3} y^{2}+a_{10} x y^{4}+a_{11} y^{5}
$$

Proof of Theorem 1.4. Let $C$ be the singular model given by Lemma 3.1. Denote by $H$ the Hasse-Witt matrix of $X$ given by Proposition 3.2 and by $e_{i}=$ $\left(e_{i, 1}, \ldots, e_{i, 5}\right)$ the $i$-th row of $H$. Then we have $\operatorname{rank}(H) \geq 1$ because of the Ekedahl's genus bound for superspecial curve [5]. Suppose $\operatorname{rank}(H)=1$. We consider different cases for the singular point of $C$.

If the curve $C$ has a node, by Lemma 3.4, $f$ is given by (14). If at least one of the $b_{0}, b_{1}$ is non-zero, by symmetry we may assume $b_{0} \neq 0$. By scaling we may assume $b_{0}=1$. Moreover, by Proposition 3.2, we have $e_{4}=\left(2 b_{2}, 0,2, b_{2}^{2}+2 b_{3}+2 a_{2}, b_{2}+2 a_{1}\right)$ which is non-zero. Then $e_{i}=\lambda_{i} e_{4}$ with $\lambda_{i} \in k$ for $i=1,2,3,5$. In particular, we have

$$
e_{5}=\left(0,2 b_{3}, 2 b_{1}, 2 b_{1} b_{3}+2 a_{5}, 2 b_{1} b_{2}+b_{3}^{2}+2 a_{4}\right)=\lambda_{5} e_{4}
$$

This implies that $b_{3}=0$ and $b_{1} b_{2}=0$.

If $b_{1}=0$, then $e_{5}=\left(0,0,0,2 a_{5}, 2 a_{4}\right)$ is the zero vector. Hence $a_{4}=a_{5}=0$. Note that in this case we have $e_{3,1}=2 a_{11}$ and $e_{3,1}=\lambda_{3} e_{4,1}=0$. Then $a_{11}=0$ and

$$
F=x y z^{3}+\left(x^{3}+b_{2} x^{2} y\right) z^{2}+\left(a_{1} x^{4}+a_{2} x^{3} y+a_{3} x^{2} y^{2}\right) z+a_{6} x^{5}+a_{8} x^{3} y^{2}+a_{9} x^{2} y^{3} \text {. }
$$

One can easily check that $(0: 0: 1)$ and $(0: 1: 0)$ are common zeros of $F=F_{x}=F_{y}=$ $F_{z}=0$. Then $C$ has at least two singular points, a contradiction.

Now if $b_{1} \neq 0$, then the equation $b_{1} b_{2}=0$ implies $b_{2}=0$. Consider a change of coordinate $x \mapsto \alpha x, y \mapsto \beta y$ and multiply $F$ by $1 /(\alpha \beta)$. The coefficients of $x^{3}$ and $y^{3}$ in $F$ become $\alpha^{2} / \beta$ and $\beta^{2} b_{1} / \alpha$. By taking $\beta=\alpha^{2}$ and $\alpha=b_{1}^{-1 / 3}$, we may assume $b_{0}=b_{1}=1$. Then $e_{5}=\lambda_{5} e_{4}$ implies $a_{2}=a_{5}$ and $a_{1}=a_{4}$. Additionally, we have

$$
\begin{aligned}
& e_{1}=\left(2 a_{1} a_{3}+a_{2}^{2}+2 a_{8}, a_{1}^{2}+2 a_{6}, 2 a_{1} a_{2}, 2 a_{1} a_{8}+2 a_{3} a_{6}, 2 a_{2} a_{6}\right), \\
& e_{2}=\left(a_{2}^{2}+2 a_{11}, a_{1}^{2}+2 a_{2} a_{3}+2 a_{9}, 2 a_{1} a_{2}, 2 a_{1} a_{11}, 2 a_{2} a_{9}+2 a_{3} a_{11}\right) .
\end{aligned}
$$

Then by $e_{1}=\lambda_{1} e_{4}$ and $e_{2}=\lambda_{2} e_{4}$, we have

$$
a_{6}=a_{1}^{2}, \quad a_{8}=a_{2}^{2}-a_{1} a_{3}, \quad a_{9}=a_{1}^{2}-a_{2} a_{3}, \quad a_{11}=a_{2}^{2} .
$$


If $a_{3}=0$, then one can easily show that $(-1: 1: 0)$ and $(0: 0: 1)$ are common zeros of $F=F_{x}=F_{y}=F_{z}=0$ and hence are singular points of $C$, a contradiction. If $a_{3} \neq 0$, then $F_{z}=2\left(x^{3}+y^{3}\right) z+a_{1} x^{4}+a_{2} x^{3} y+a_{3} x^{2} y^{2}+a_{1} x y^{3}+a_{2} y^{4}$ and by substituting

$$
z=\left(a_{1} x^{4}+a_{2} x^{3} y+a_{3} x^{2} y^{2}+a_{1} x y^{3}+a_{2} y^{4}\right) /(x+y)^{3}
$$

in $F_{x}$ and $F_{y}$ and by letting $y=1$, we have $\left.\left(x(x+y)^{9} F_{x}\right)\right|_{y=1}=\left.\left((x+y)^{9} F_{y}\right)\right|_{y=1}$ and

$$
\begin{aligned}
\left.\left((x+y){ }^{9} F_{x}\right)\right|_{y=1} & =\left(a_{1}^{3}+a_{1} a_{2}\right) x^{12}+\left(a_{1} a_{2}+a_{2}^{3}+2 a_{3}^{2}\right) x^{9}+\left(a_{3}^{3}+a_{3}^{2}\right) x^{6} \\
& +\left(a_{1}^{3}+a_{1} a_{2}+2 a_{3}^{2}\right) x^{3}+a_{1} a_{2}+a_{2}^{3} .
\end{aligned}
$$

Since $a_{3} \neq 0$, one can check that it has solutions with $x \neq-1,0$. Then there exists another singular point on $C$ which is distinct from $(0: 0: 1)$, a contradiction.

Now if $b_{0}=b_{1}=0$, we have $e_{4}=\left(2 b_{2}, 0,0, b_{2}^{2}+2 a_{2}, 2 a_{1}\right)$ and $e_{5}=\left(0,2 b_{3}, 0,2 a_{5}, b_{3}^{2}+\right.$ $\left.2 a_{4}\right)$. Since $\operatorname{rank}(H)=1$, we have at least one of the $b_{2}, b_{3}$ is zero. By symmetry we may assume $b_{3}=0$. If $b_{2} \neq 0$, by scaling we may assume $b_{2}=1$. Note that we have $e_{i}=$ $\lambda_{i} e_{4}$ with $\lambda_{i} \in k$ for $i=1,2,3,5$. In particular for $i=5$, it is straightforward to see that $\lambda_{5}=0$ and $a_{4}=a_{5}=0$. Moreover, $e_{2,2}=2 a_{11}=\lambda_{2} e_{4,2}=0$. Then by a similar fashion, one can show that $(0: 0: 1)$ and $(0: 1: 0)$ are singular points of $C$, a contradiction. If $b_{3}=b_{2}=0$, then

$$
e_{1}=\left(2 a_{1} a_{3}+a_{2}^{2}, a_{1}^{2}, 2 a_{1} a_{2}, 2 a_{1} a_{8}+2 a_{3} a_{6}, 2 a_{2} a_{6}\right), \quad e_{4}=\left(0,0,0,2 a_{2}, 2 a_{1}\right) .
$$

This implies $a_{1}=a_{2}=0$ otherwise $e_{1}$ and $e_{4}$ are linearly independent. Similarly one has $a_{4}=a_{5}=0$ by checking the linearly independence of $e_{2}$ and $e_{5}$. Now we have $e_{4}=e_{5}=0$ and

$$
e_{1}=\left(0,0,0,2 a_{3} a_{6}, 0\right), \quad e_{2}=\left(0,0,0,0,2 a_{3} a_{11}\right), \quad e_{3}=\left(0,0, a_{3}, 2 a_{3} a_{9}, 2 a_{3} a_{8}\right) .
$$

Since $\operatorname{rank}(H)=1$, we obtain that $a_{6}=a_{11}=0$ and $F=y\left(x z^{3}+a_{3} x^{2} y z+a_{8} x^{3} y+\right.$ $\left.a_{9} x^{2} y^{2}\right)$, a contradiction.

Now if $C$ has a cusp, then by Lemma 3.4, $f$ is given by (15). Moreover, by Proposition 3.2, we have

$$
e_{4}=\left(2 b_{3}, 0,2 b_{2}, b_{2}^{2}+2 a_{3}, 2 a_{2}\right), \quad e_{5}=\left(0,2,0,2 b_{3}, 2 b_{2}+b_{3}^{2}+2 a_{5}\right) .
$$

If $\operatorname{rank}(H)=1$, then $e_{i}=\lambda_{i} e_{5}$ with $\lambda_{i} \in k$ for $i=1,2,3,4$. In particular, $e_{4}=\lambda_{4} e_{5}$ implies $\lambda_{4}=b_{2}=b_{3}=a_{2}=a_{3}=0$. Then we obtain

$$
\begin{aligned}
& e_{1}=\left(0, a_{1}^{2}, 0,2 a_{1} a_{8}, 2 a_{1} a_{7}\right), \\
& e_{2}=\left(a_{5}^{2}+2 a_{11}, a_{4}^{2}, 2 a_{4} a_{5}+2 a_{10}, 2 a_{4} a_{11}+2 a_{5} a_{10}, 2 a_{4} a_{10}\right), \\
& e_{3}=\left(2 a_{8}, 2 a_{1} a_{4}, 2 a_{1} a_{5}+2 a_{7}, 2 a_{1} a_{11}+2 a_{4} a_{8}+2 a_{5} a_{7}, 2 a_{1} a_{10}+2 a_{4} a_{7}\right) .
\end{aligned}
$$


Hence by $e_{i}=\lambda_{i} e_{5}$ for $i=1,2,3$, we get $a_{7}=2 a_{1} a_{5}, a_{8}=0, a_{10}=2 a_{4} a_{5}, a_{11}=a_{5}^{2}$. Additionally, one can easily check that $F=F_{x}=F_{y}=F_{z}=0$ have common zeros $(0: 0: 1),(0$ : $1: 0)$ if $a_{5}=0$ and $(0: 0: 1),\left(0: 1 /\left(2 a_{5}\right): 1\right)$ if $a_{5} \neq 0$, a contradiction. Then $\operatorname{rank}(H) \geq 2$ if $C$ has a cusp.

In any case, we have $\operatorname{rank}(H) \geq 2$ and hence $a_{X} \leq 3$.

Acknowledgments. I would like to thank my supervisor, Professor Gerard van der Geer, for his patient and continuous guidance and kind advice throughout my research studies. I also would like to thank Katsura and Pries for helpful comments.

\section{References}

1. Arbarello, E., Cornalba, M., Griffiths, P. A. and Harris, J., Geometry of algebraic curves. Vol. I, Grundlehren der Mathematischen Wissenschaften. 267, Springer, New York, 1985.

2. Booher, J. and Bryden, binitsCais, a-numbers in Artin-Schreier covers, Preprint, 2018.

3. Cartier, P., Une nouvelle opération sur les formes différentielles, C. R. Acad. Sci. Paris 244 (1957), 426-428.

4. Chai, C. and Oort, F., Monodromy and irreducibility of leaves, Ann. Math. 173 (2011), 1359-1396.

5. Ekedahl, T., On supersingular curves and abelian varieties, Math. Scand. 60 (1987), $151-178$.

6. Ekedahl, T. and van der Geer, G., Cycle Classes of the E-O Stratification on the Moduli of Abelian Varieties, in Algebra, Arithmetic, and Geometry: Volume I: In Honor of Yu. I. Manin, pp. 567-636, Birkhäuser, Boston, 2009.

7. Elkin, A. and PRIES, R., Ekedahl-Oort strata of hyperelliptic curves in characteristic 2, Algebra Number Theory 7 (2013), 507-532.

8. Farnell, S. and Pries, R., Families of Artin-Schreier curves with Cartier-Manin matrix of constant rank, Linear Algebra Appl. 439 (2013), 2158-2166.

9. IrokaWA, S. and SASAKi, R., A remark on Artin-Schreier curves whose Hasse-Witt maps are the zero maps, Tsukuba J. Math. 15 (1991), 185-192.

10. Kudo, M. and Harashita, S., Superspecial trigonal curves of genus 5. Preprint, 2018, arXiv:1804.11277

11. OdA, T., The first de Rham cohomology group and Dieudonné modules, Ann. Sci. Éc. Norm. Supér. 4 (1969), 63-135.

12. Oort, F., A Stratification of a Moduli Space of Polarized Abelian Varieties in Positive Characteristic, in Moduli of Curves and Abelian Varieties: The Dutch Intercity Seminar on Moduli, pp. 47-64, Vieweg+Teubner Verlag, Wiesbaden, 1999.

13. Re, R., The rank of the Cartier operator and linear systems on curves, J. Algebra 236 (2001), 80-92.

14. Seshadri, C. S. L'opération de Cartier. Applications, Sémin. Claude Chevalley 4 (1958-1959), 1-26. 
15. Stöhr, K. and Voloch, J., A formula for the Cartier operator on plane algebraic curves, J. Reine Angew. Math. 377 (1987), 49-64.

16. Subrao, D., The p-rank of Artin-Schreier curves, Manuscr. Math. 16 (1975), 169-193.

17. Sullivan, F. J., p-torsion in the class group of curves with too many automorphisms, Arch. Math. (Basel) 26 (1975), 253-261.

18. TAIT, J., Group actions on differentials of curves and cohomology bases of hyperelliptic curves, Ph.D. Thesis, University of Southampton, 2014.

\author{
Zijian Zhou \\ College of Liberal Arts and Sciences \\ National University of Defense Technology \\ 410073 Changsha \\ China \\ zhouzijian.edu@gmail.com
}

Received November 25, 2019

in revised form September 27, 2020 